\title{
Stocking density and its influence on shape of Senegalese sole adults
}

\author{
Pedro Pablo Ambrosio ${ }^{1,2, *}$, Corrado Costa ${ }^{3}$, Pablo Sánchez ${ }^{1,2}$, Rosa Flos ${ }^{1,2}$ \\ ${ }^{1}$ Department of Agri-food Engineering and Biotechnology, Technical University of Catalonia (UPC). Av. Canal Olímpic, \\ $s / n .08860$ Castelldefels. Barcelona, Spain. \\ ${ }^{2}$ Centre de Referència de Recerca i Desenvolupament en Aqüicultura de la Generalitat de Catalunya. \\ ${ }^{3}$ Agritech Lab CRA-I.S.M.A. (Council for Research in Agriculture - Agricultural Mechanization Research Institute). Via \\ della Pascolare, 16, 00016 Monterotondo (Rome), Italy. \\ ${ }^{*}$ Author for correspondence (e-mail: pepambros@telefonica.net; phone: +34 935521056)
}

\begin{abstract}
Morphological changes in Senegalese sole adults (Solea senegalensis; Kaup 1858) reared at two stocking density conditions (low stocking density, $60 \%$ of bottom occupation and high stocking density, $180 \%$ of bottom occupation) were investigated using geometric morphometrics. Canonical variate analysis on weight matrix scores, including the uniform component $\left(\mathrm{W}^{\prime}\right)$, at the end of the experiment revealed differences in body shape between experimental groups. Low density group presented a similar change pattern to High density group but less intense. Differences were discussed in terms of the effect of a high stocking density on the shape of soles which were very close to commercialization. Results did not indicate a significant size-related shape, likely due to fish age.

Our results provide a first promising insight in the study of environment-related shape changes in reared sole.
\end{abstract}

Keywords: Solea senegalensis, geometric morphometrics, shape, stocking density.

\section{Introduction}

Marine aquaculture, specially producers from southern Europe, are showing interest in a promising species, Senegalese sole (Solea senegalensis; Kaup, 1858). Sole has both a good commercial assessment and a very good consumer approval.

Despite sole is an attractive candidate for marine aquaculture, production parameters in all rearing stages need to be optimized to manage an industrial level (Imsland 2003). Among production parameters to be optimized, stocking density has capital importance as much as for its consequences on growth performance as for its likely effect on fish shape.

Originally Published as: Ambrosio, P.P., Costa, C., Sánchez, P., Flos, R. Stocking density and its influence on shape of Senegalese sole adults. Aquaculture International IN PRESS. DOI 10.1007/s10499-007-9147-5 http://www.springerlink.com/content/9238065103w38371/fulltext.pdf 
Several studies have investigated effect of stocking density on growth performance of soles. Howell (1998) working with S. solea less than $15 \mathrm{~g}$ and Schram et al. (2006) with fish from 35 to $40 \mathrm{~g}$ have reported a significant decreasing of growth with high stocking densities. Engrola et al. (2001) found that growth was scarcely affected by stocking density (up to $4.5 \mathrm{~kg} / \mathrm{m}^{2}$ ) while rearing juveniles of $S$. senegalensis ranging from 10 to $25 \mathrm{~g}$. More recently, we found that individual growth parameters of adults with an initial mean weight of $300 \mathrm{~g}$ were only negatively affected by stocking density ( $180 \%$ of bottom occupation, $26 \mathrm{Kg} / \mathrm{m}^{2}$ ) for the first 60 days of experience (unpublished data).

The effects of rearing conditions on fish shape are important for aquaculture, given that a shape considered as 'strange' by consumer could cause a deal of harm to their perception of fish as a product. From early dates (Hubss 1926; Barlow 1961) many reports have been issued describing how fish form can be affected by environmental parameters (such as salinity, temperature, etc.) when fish moves into a new environment. Accordingly, Corti et al. (1996) found significant differences in sea bass (Dicentrarchus labrax) shape after its acclimation to freshwater. From an anatomical point of view, external shape is related to internal skeletal anomalies (Loy et al. 2000). Likewise, Loy et al. (1999) demonstrated that the effects of different larval and postlarval rearing condition on external morphology could be detected in juveniles and adults of sea bream (Sparus aurata).

Form change has been studied in several animal species using geometric morphometrics (Bookstein 1991; Rohlf and Bookstein 1991; Rohlf and Marcus 1993; Marcus et al. 1993). Geometric morphometric methodology implies multivariate analysis of landmark coordinates located following certain rules on the surface of a morphological object. The morphological object or specimen is described in the space by a set of landmarks, $X$ and $Y$ coordinates, which are homologous and can be recovered unambiguously from specimen to specimen (Bookstein 1991). Each individual is then described by a landmark configuration. There are two principal methods of shape comparisons in geometric morphometric, the Procustes superimposition and thin-plate spline analysis. In the first case shape changes are described after superimposition individuals one over the other according to a fitting criteria. Superimposition implies translation and rotation based on generalized least square method (Rolhf and Slice 1990). From the superimposed configuration, a mean configuration (consensus) is obtained and used as reference. All configurations are scaled according to a size measured known as centroid size. Centroid size is removed from the analysis of shape (Loy et al. 2000). Procustes residuals are the outcome shape variables which remain after isometric alignment of the shapes being compared. Their summation over all landmarks yields Procustes distances among these shapes. The Procustes distances can be used in multivariate analyses just as the Euclidean distances (Pavlinov 2002). 
Thin-plate spline is an interpolating function (Bookstein 1991), that uses residuals after fitting shapes to the consensus by stretching/compressing and shearing until complete identity of their landmark configuration, to compute a new set of variables (partial warps and relative warps) that can be analyzed with multivariate techniques. Shapes differences can be visualized as deformation grid which displays type, amount and localization of shape differences.

As far as we know, no studies have been conducted to determine whether stocking density has an effect on sole shape. Therefore, the aim of this work was study a likely effect of stocking density on shape of Senegalese sole adults.

\section{Material and Methods}

Fish, facilities and experimental design.

A total of ninety six $S$. senegalensis adults $(318.7 \pm 7.8 \mathrm{~g})$ were used. Fish coming from the same broodstock were obtained from a Mediterranean commercial farm (Tarragona, Spain), and were acclimatized for more than 60 days at the Mediterranean Marine and Environmental Research Center (CMIMA) in Barcelona, Spain.

The experiment was performed at a flow-through water system facility where main water parameters were controlled. Temperature, dissolved oxygen, salinity, and water renovation were daily monitored ( $\mathrm{T}=20 \pm 1 \stackrel{\circ}{\circ} \mathrm{C}$; Oxygen $>60 \%$ of saturation; Salinity= $38.2 \mathrm{~g} / \mathrm{l}$; water renovation $30 \%$ per hour). Total dissolved ammonia- $\mathrm{N}$ was checked twice, at the beginning and at the end of the experiment, and no dangerous level was detected. Tanks were routinely inspected for mortalities and cleaning. Photoperiod for latitude 41 oN from July to November with artificial dusk and dawn was simulated with fluorescent light dimmed by shading covers laid over the tanks.

Fish were fed overnight by means of a programmable electronic feeder. Feeding was scheduled in four feed takes spread from dusk to dawn. Daily ration was set ad libitum, after it had being optimized during the conditioning period in order to minimize uneaten feed in the tank. A commercial feed for sole (ProAqua-Solea \#3) was used.

In order to set a triplicate experimental layout, 3 square section tanks of $0.88 \mathrm{~m}^{2}$ of bottom surface and a volume of 700 I were equipped with a set of 2 movable and adjustable dividers that provided 3 rectangular section volumes in each tank: two main volumes for both a high density treatment and a control treatment and a third buffer volume among them, conforming a total of 6 experimental units of variable bottom surface. The dividers consisted in a PVC frame tightly adjusted to the walls of the tank holding a plastic net that allowed the free circulation of water throughout all the water volume, while keeping fish confined to their 
assigned experimental unit.

Fish were randomly distributed into two different stocking density experimental groups: a low density group, which was considered the control group ( $C ; N=48,16$ fish per replicate), where stocking density was $60 \%$ of bottom occupation $\left(8.6 \pm 0,24 \mathrm{~kg} / \mathrm{m}^{2}\right)$, and a high density group $(\mathrm{H} ; \mathrm{N}=48,16$ fish per replicate), with a stocking density of $180 \%$ of bottom occupation (26.6 \pm $0,13 \mathrm{~kg} / \mathrm{m}^{2}$ ). Fish density was controlled altering the available bottom surface by displacing the movable dividers. The buffer volume between experimental units was designed to absorb this movement when mortality or growth altered the nominal density set at the beginning of the experiment and obliged us to correct the surface of each separation, thus keeping density constant all along the experiment.

At a certain point of the experiment (day 77), fish size in both treatments was too large to keep the initial density because of tank size constraints, so the available surface settings were then fixed and stocking densities increased proportionally as fish grew up.

The experimental period lasted 126 days during which fish were sampled five times. On the first biometry, fish were anaesthetized (MS 222, $200 \mathrm{mg} / \mathrm{ml}$ in sea water bath until apparent signals of sedation. Bath time varied according to weight, with a mean value of $9 \mathrm{~min}$ ) and then individually tagged by means of an intradermal injection of ink (Reig et al. 2003). Afterwards, fish were measured, weighted and photographed. This procedure was repeated for subsequent samplings, excluding the tagging protocol.

On each biometry day, digital images (HP Photosmart 850. Pictures with a resolution of $1600 \times 1200$ pixels) of individual fish were collected by placing the specimens properly identified on a plastic graph paper sheet. Photographic plane was oriented parallel to the ocular side of soles. This setting helped to correct possible slight differences in focal distance among fish images. Fish images showing apparent skeletal malformations were not taken into account to geometric morphometrics analysis.

\section{Geometric morphometrics}

After studying skeleton by means of double-stain method (Taylor and Van Dyke 1985; Gavaia et al. 1999), a total of 20 landmarks were identified and then digitalized using the software TPS Digit (Rohlf, 2004a), applied on the ocular side of each specimen (Figure 1).

Coordinates were superimposed by means of the Generalized Procustes Analysis (GPA). This preserves all information about shape removing only the information unrelated to shape (scale, position and orientation) (Rohlf and Slice 1990). A consensus configuration was computed.

Centroid size (CS), defined as the square root of the squared distance between each landmark 
and the centroid of the landmark configurations summed over all landmarks, was also computed and used as independent size variable. GPA and CS calculations were done in TPSregr software (Rohlf 2003).

Relationship between shape and size was assessed computing Procustes distances from specimen to the consensus configuration and from each specimen to the configurations predicted by linear regression. A generalization of Goodall's F-ratio (Goodall 1991) was used to evaluate the fit of the regression model. Permutation test for Wilks' Lambda was also performed.

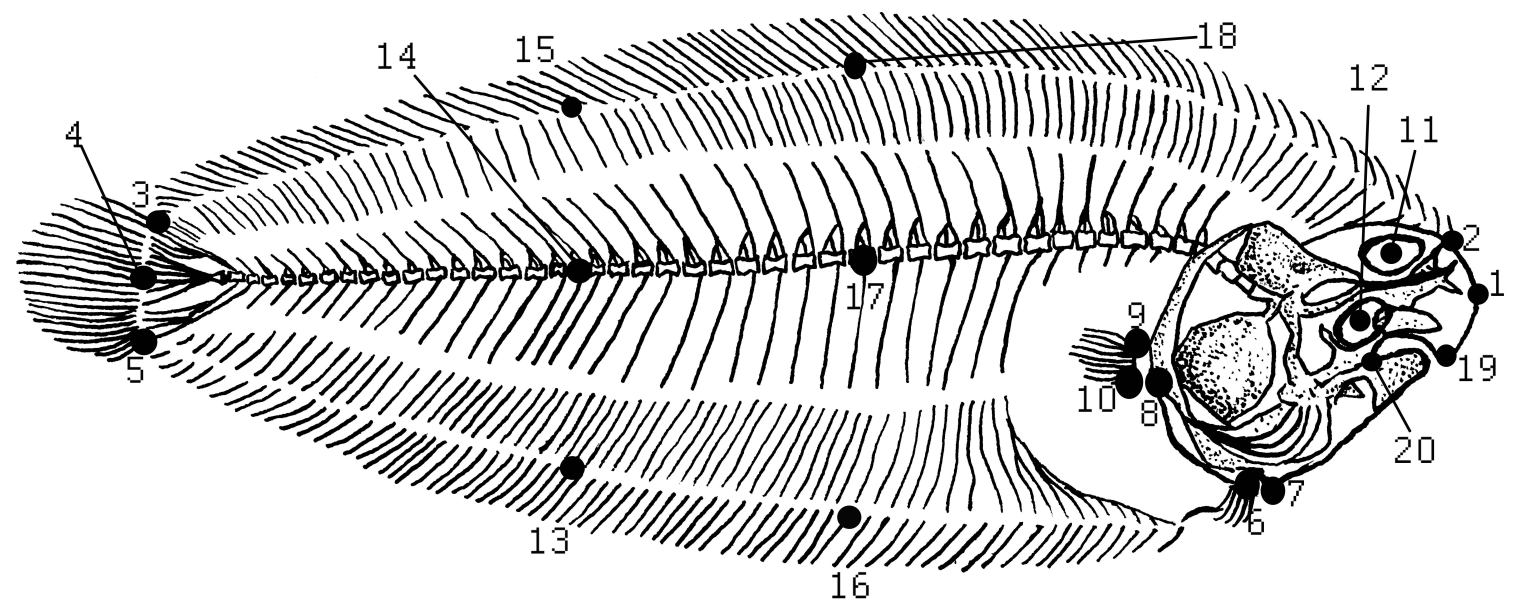

Fig. 1. Landmarks collected on Senegalese sole. 1) Snout tip; 2) anterior origin of the dorsal fin; 3 ) dorsal origin of the caudal fin; 4) caudal end of lateral line, 5) ventral origin of the caudal fin; 6) origin of the pelvic fin; 7) insertion of operculum on the ventral profile; 8 ) end of operculum; 9) upper side of the pectoral fin; 10) lower side of the pectoral fin; 11) center of the left ocular space; 12) center of the right ocular space; 13) insertion of the 30th anal fin ray (counted from posterior insertion of the anal fin); 14 ) intersection between the projection from the 30th anal fin ray perpendicularly to the major axis (axis between landmark 1 and 4) and the lateral line; 15) intersection between the projection from the 30th anal fin ray perpendicularly to the major axis (axis between landmark 1 and 4 ) and the dorsal profile; 16) insertion of the 50th anal fin ray (counted from posterior insertion of the anal fin); 17) intersection between the projection from the 50th anal fin ray perpendicularly to the major axis (axis between landmark 1 and 4 ) and the lateral line; 18 ) intersection between the projection from the 50th anal fin ray perpendicular to the major axis (axis between landmark 1 and 4) and the dorsal profile; 19) origin of the mouth (upper jaw); 20) end of the mouth (lower jaw). The figure represents an illustration of the skeleton of Solea senegalensis adult, including the outline of eyes (non osseous structures).

In order to describe shape differences between experimental groups, a canonical variate analysis (CVA) (SAS, v 8.0) was performed using weight matrix scores including the uniform component $\left(W^{\prime}\right)$. Splines characteristics of each shape were used to visualize and describe shape differences (TPSRelw software, Rohlf 2004b; data not shown). CVA was performed in order to simplify the description of differences among group means. CVA constructs a new coordinate system (the canonical variates, CVs) and determines the scores on those axes for all individuals in a study. Also, CVs are linear combination of the original variables and are 
constrained to be mutually orthogonal. CVA uses the patterns of within-group variation to scale axes of the new coordinate system. Because of this rescaling, CVs are not simply rotations of the original coordinate system, and distances in CV space are not equal to distances in the original coordinate system. As result of the rescaling, CV1 is the direction in which groups are most effective discriminated (Zelditch et al. 2004)

Previously to the CVA, multivariate normality (Mardia's multivariate test -PAST $\mathrm{v} 1.74$ software), homoscedasticity (modified Barlett's test -SAS software v. 8.0) and multivariate linearity (as suggested by Zelditch et al., 2004 -SAS software $v$ 8.0) were tested.

A multivariate contrast to separate groups was performed. Mahalanobis distances were also computed and tested.

In order to describe possible influences of sex on shape, a CVA was performed taking into account males and females at the beginning of the experiment, males and females reared under high stocking density at the end of the experiment and males and females reared under low stocking density at the end of the experiment.

\section{Results}

Thirty days after the beginning of the experiment one replicate of each experimental group had to be removed due to a disease outbreak.

Initial and final stocking density, as well as initial and final mean weight, and mean specific growth rate (SGR), for both control and high density treatments are shown in table 1.

Table 1. Mean values ( \pm SEM ${ }^{a}$ ) of initial and final weight, initial and final stocking density and SGR of Senegalese sole reared at two different stocking densities for 126 days.

\begin{tabular}{llllll}
\hline $\mathrm{SD}^{\mathrm{b}}$ & $\begin{array}{l}\text { Initial Density } \\
\left(\mathrm{kg} / \mathrm{m}^{2}\right)\end{array}$ & $\begin{array}{l}\text { Final Density } \\
\left(\mathrm{kg} / \mathrm{m}^{2}\right)\end{array}$ & $\begin{array}{l}\text { Initial weight } \\
(\mathrm{g})^{\mathrm{c}}\end{array}$ & $\begin{array}{l}\text { Final weight } \\
(\mathrm{g})^{\mathrm{c}}\end{array}$ & $\mathrm{SGR}^{\mathrm{c}}$ \\
\hline $\mathrm{C}$ & $8,6 \pm 0,17$ & $11,5 \pm 0,13$ & $317,0 \pm 17,67^{\mathrm{ns}}$ & $495,9 \pm 28,48^{\mathrm{ns}}$ & $0,35 \pm 0,020^{*}$ \\
$\mathrm{n}$ & 31 & 19 & 19 & 19 & 19 \\
\hline $\mathrm{H}$ & $26,6 \pm 0,09$ & $39,8 \pm 0,54$ & $353,0 \pm 14,67^{\mathrm{ns}}$ & $481,7 \pm 19,56^{\mathrm{ns}}$ & $0,25 \pm 0,014^{*}$ \\
$\mathrm{n}$ & 31 & 25 & 25 & 25 & 25 \\
\hline
\end{tabular}

\footnotetext{
a SEM, standard error of the mean

b SD, Stocking density group. C, control group (stocking density of $60 \%$ of bottom occupation). H, high stocking density group (180\% of bottom occupation).

${ }^{c}$ Mean values noted with * indicate signifficant differences between groups (ANOVA, $P<0.001$ ).
} 
Table 2. Mean values $\left( \pm \mathrm{SEM}^{\mathrm{a}}\right.$ ) of centroid size (CS) of Senegalese sole reared at two different stocking densities.

\begin{tabular}{|c|c|c|c|c|c|}
\hline \multirow[t]{2}{*}{$\mathrm{SD}^{\mathrm{b}}$} & \multicolumn{5}{|c|}{ CS mean value $(\mathrm{cm})$} \\
\hline & Biometry $1^{\mathrm{c}}$ & Biometry $2^{c}$ & Biometry $3^{c}$ & Biometry $4^{c}$ & Biometry $5^{c}$ \\
\hline C & $41.7 \pm 0.58 \mathrm{~ns}$ & $42.6 \pm 0.87^{\mathrm{ns}}$ & $44.5 \pm 0.90^{\mathrm{ns}}$ & $45.7 \pm 0.77^{\mathrm{ns}}$ & $46.7 \pm 0.95^{\mathrm{ns}}$ \\
\hline$n^{d}$ & 31 & 19 & 19 & 19 & 19 \\
\hline $\mathrm{H}$ & $41.7 \pm 0.58^{\mathrm{ns}}$ & $42.5 \pm 0.67^{\mathrm{ns}}$ & $45 \pm 0.61^{\text {ns }}$ & $44.9 \pm 0.64^{\mathrm{ns}}$ & $46.5 \pm 0.66^{\mathrm{ns}}$ \\
\hline$n^{d}$ & 31 & 25 & 25 & 25 & 25 \\
\hline
\end{tabular}

\footnotetext{
a SEM, standard error of the mean density group (180\% of bottom occupation).

c Mean values noted with ns did not differ significantly statistically between groups $(P<0.05)$.

a Number of images collected and digitalized per biometry.
}

b SD, Stocking density group. C, control group (stocking density of $60 \%$ of bottom occupation). H, high stocking

Experimental groups shown similar mean sizes (mean centroid size) along the experiment, therefore no significant differences in size between experimental groups were found in any of the five biometries (Table 2).

Shape and size relationship

A general significant lineal relationship between shape and size was found when this relationship was assessed by regression of partial warps on CS and when it was assessed from regression of Procustes distances on CS (except for non uniform component of high density group, Goodall's test, $\mathrm{p}=0.22$; Table 3 ).

Table 3. Significance probability after multivariate linear regression of each partial warp and the uniform shape component on centroid size and significance probability of Goodall's test after linear regression of Procustes distances on centroid size.

\begin{tabular}{llll}
\hline Group $^{\mathrm{a}}$ & Shape component & Multivariate test SP & $\begin{array}{l}\text { Goodall's test } \\
\mathrm{SP}^{\mathrm{b}}\end{array}$ \\
\hline $\mathrm{C}$ & Uniform + non uniform & $2.810^{-9}$ & $<0.0001$ \\
& Non uniform & $1.210^{-9}$ & $<0.0001$ \\
$\mathrm{H}$ & Uniform + non uniform & $1.010^{-9}$ & 0.002 \\
& Non uniform & $3.510^{-9}$ & 0.22 \\
\hline
\end{tabular}

${ }^{a} \mathrm{C}$, control group (stocking density of $60 \%$ of bottom occupation). $\mathrm{H}$, high stocking density group (180\% of bottom occupation).

${ }^{b}$ SP, significance probability. 
Nevertheless, regression coefficients of partial warps and uniform components on CS were very low in both groups (less than 0.18 ) showing a poor relationship between each shape variable and size after lineal fitting. The low percentage of data variance explained by the linear regression of Procustes distances on CS (3.8 \% and $1.3 \%$ for $\mathrm{C}$ and $\mathrm{H}$ respectively) also points towards the same conclusion, revealing a weak link between size and shape during the experimental period. Focusing on this low percentage of shape variability explained by size, differences between groups in size contribution on the uniform and non-uniform shape changes were found. In $\mathrm{C}$, the change of the uniform component of shape was hardly affected by size. However in $\mathrm{H}$, size increment had a moderate contribution on uniform component (Table 4).

Finally, regression of relative warps on CS confirmed the lack of significant relationships between size and shape. Figure $2 \mathrm{a}$ and $2 \mathrm{~b}$ describe shape variability along relative warp 1 , showing that shape variability was not correlated with CS $(r=0.19$ for control and $r=0.01$ for high density).

\section{Shape differences.}

Canonical Variate Analysis (CVA) revealed differences in body shape. Specifically, C and H groups could be separated between them and from initial shape using scores on CV1 and CV2 (Fig. 3a).
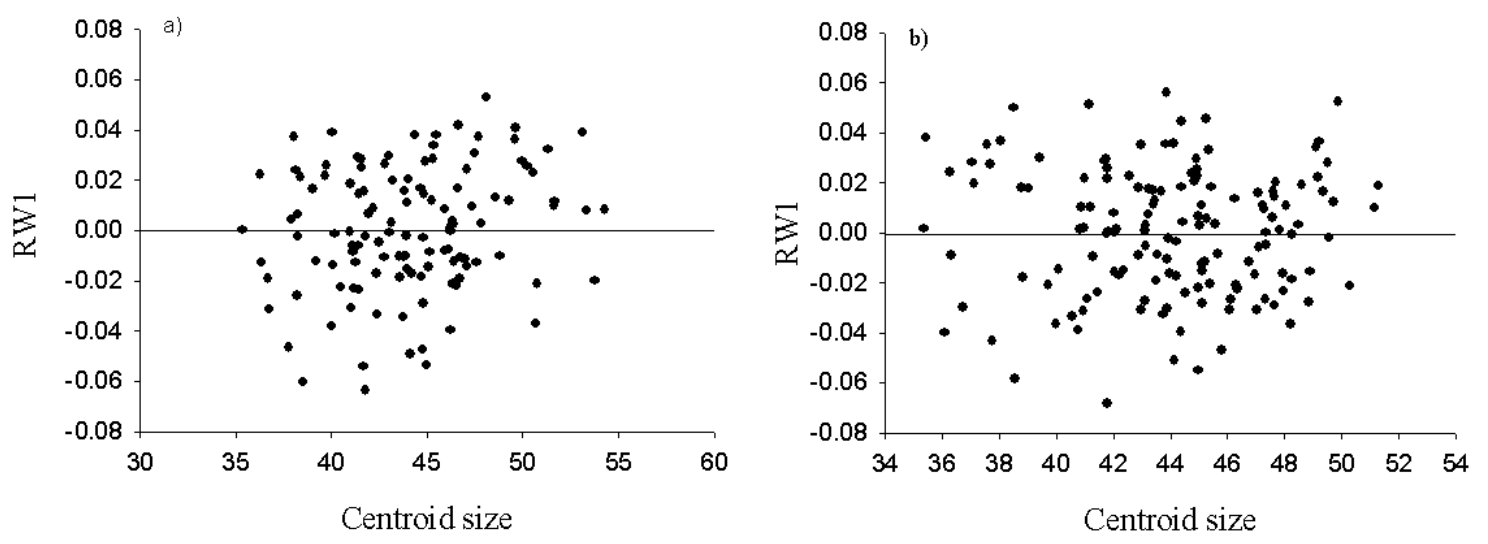

Fig. 2. Shape variability as summarized by relative warp 1 (RW1) in a) Control group with a stocking density of $60 \%$ of bottom occupation and in b) High density group with a stocking density of $180 \%$ of bottom occupation 
Table 4. Percentages of the variance explained after lineal regression of Procustes distances on centroid size.

\begin{tabular}{lllll}
\hline & $\mathrm{PCS}^{\mathrm{a}}$ & $\mathrm{CNU}^{\mathrm{b}}$ & $\mathrm{PNU}^{\mathrm{c}}$ & $\mathrm{PU}^{\mathrm{d}}$ \\
\hline Control group & 3.8 & 3.5 & 91.2 & 8.8 \\
High density group & 1.2 & 0.8 & 65.7 & 34.3 \\
\hline
\end{tabular}

a PCS, percentage of explained variance including both uniform and non uniform shape components.

${ }^{\mathrm{b}} \mathrm{CNU}=$ Percentage of explained variance without the uniform component.

c PNU, non uniform contribution to total variability explained by size (\%)

${ }^{\mathrm{d}} \mathrm{PU}$, uniform contribution to total variability explained by size (\%).
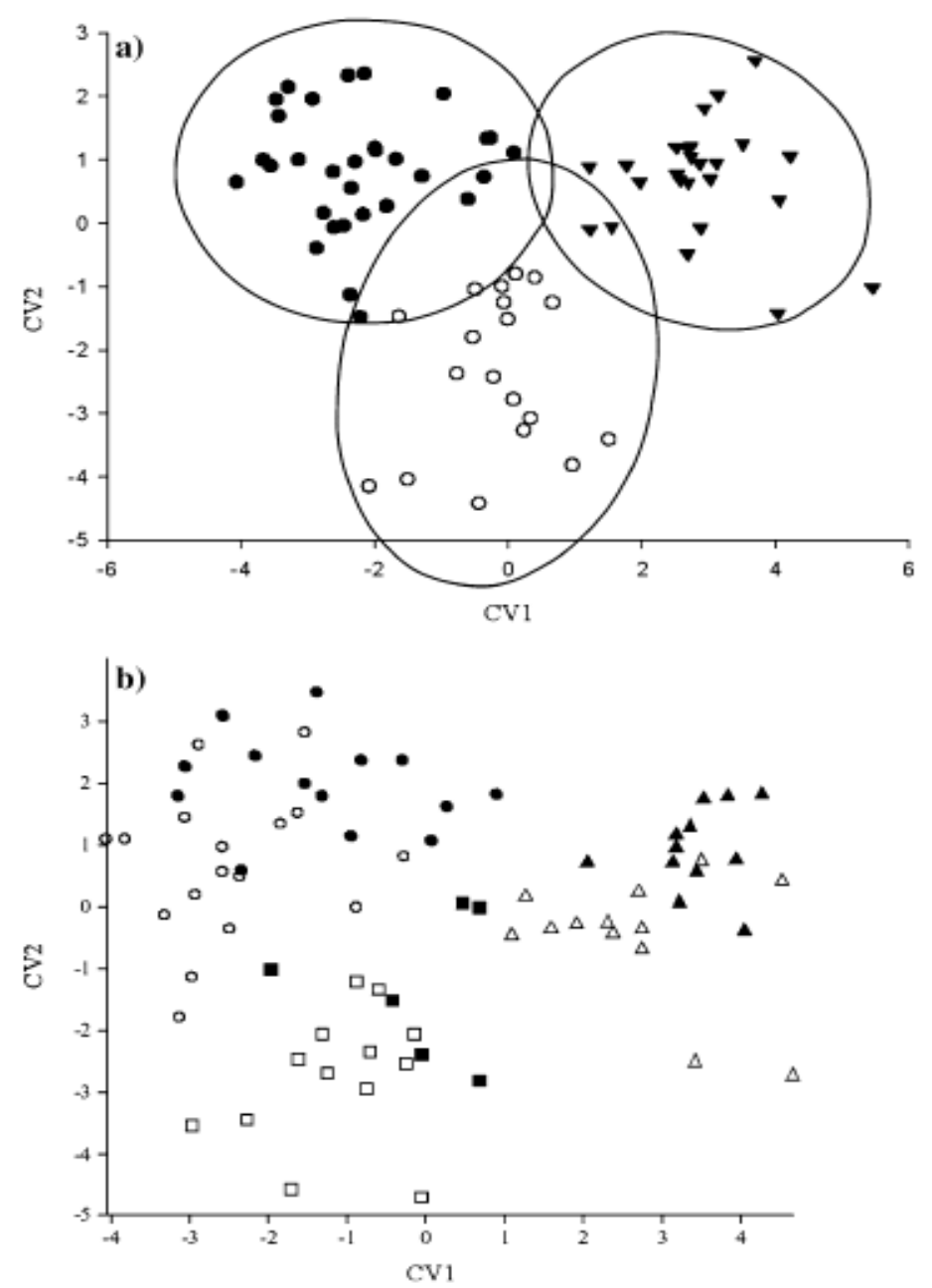

Fig. 3. a) Scatter plots from the Canonical Variate Analysis (CVA). ( - ) all fish pooled at the beginning of the experiment; $(O)$ control group fish ( $60 \%$ of bottom occupation) at the end of the experiment. ( $\boldsymbol{\nabla}$ ) high density group fish ( $180 \%$ of bottom occupation) at the end of the experiment. b) Scatter plots from the Canonical Variate Analysis (CVA) of Senegalese sole grouped by sex at the beginning of the experiment ( $\bigcirc$ : males; $\bullet$ : females ), and at the end of the experiment, also grouped by sex and stocking density (low density, 60\% of bottom occupation; $\square$ : males; females. High density, $180 \%$ of bottom occupation. $\Delta$ : males; $\mathbf{\Delta}$ : females) 
Table 5. Mahalanobis distances matrix for squared distances from the beginning of the experiment to Control and High density groups at the end of the experiment, and between them at the end of the experiment.

\begin{tabular}{llll}
\hline & $\mathrm{T}_{0}{ }^{a}$ & $\mathrm{C}_{\text {end }} \mathrm{b}^{\mathrm{b}}$ & $\mathrm{H}_{\text {end }}{ }^{\mathrm{c}}$ \\
\hline $\mathrm{T}_{0}^{\mathrm{a}}$ & 0 & 2.4186 & 5.0706 \\
$\mathrm{PS}^{\mathrm{d}}$ & - & 0.0045 & $<0.0001$ \\
$\mathrm{C}_{\text {end }}{ }^{\mathrm{b}}$ & 2.4186 & 0 & 2.8804 \\
$\mathrm{PS}^{\mathrm{d}}$ & 0.0045 & - & 0.0009 \\
$\mathrm{H}_{\text {end }}{ }^{\mathrm{a}}$ & 5.0706 & 2.8804 & 0 \\
$\mathrm{PS}^{\mathrm{d}}$ & $<0.0001$ & 0.0009 & -
\end{tabular}

a $T_{0}$, beginning of the experiment.

${ }^{b} C_{e n d}$, control group at the end of the experiment.

${ }^{c} \mathrm{H}_{\text {end }}$, high density group at the end of the experiment.

${ }^{\mathrm{d}} \mathrm{PS}$, probability of significance.

CV1 shown a higher discriminatory efficiency than CV2 (squared canonical correlation, $r^{2}=$ 0.83 and $r^{2}=0.66$ respectively). Both multivariate contrasts and Mahalanobis distances (Table 5 ) indicated significant differences between experimental groups (Wilks' lambda Prob. Sig. $>F$ $=<0.0001, \mathrm{~F}$-value $=0.05695, \mathrm{Num} . \mathrm{DF}=72$ ).

The Canonical Variate Analysis of experimental groups considering fish sex performed similar results than CVA without sex distinction. (Fig. 3b).

\section{Discussion}

Consumers often relate fish quality to appearance, odour, flavour and texture (Cato 1998). Therefore, visual perception is an important parameter for consumer approval. Environmental parameters, such as salinity can influence fish shape. Corti et al. (1996) found significant differences in sea bass shape after its acclimation to freshwater. Likewise, shape changes induced by different larval rearing conditions could be detected after 15 months of common rearing in floating cages (Loy et al. 2000). However in this study there was an important difference in initial stocking density between studied groups, $0.13 \mathrm{~kg} / \mathrm{m}^{3}$ versus $0.32 \mathrm{~kg} / \mathrm{m}^{3}$. Flos et al. (2002) studied the effects of different rearing systems on gilthead sea bream quality including an assessment of the effects on shape trough classical biometric determinations. Culture systems differed mainly in its degree of intensity, being the level of stocking density an important experimental parameter but not the unique able to influence results. Authors found that the super-intensive culture system significantly affected sea bream shape producing more compact fish that, moreover, lacked the characteristic colour pattern of this 
species.

At the end of the experiment conducted throughout our study, where stocking density was identified as the unique independent variable, significant differences in body shape between experimental groups were found. Control group showed a change pattern similar to the one presented by the high density group but less enough intense. At the end of the experiment soles reared in high stocking density presented a wider head than control fish. This change is determined by forward displacement of landmarks numbers 6 (origin of the pelvic fin) and 7 (origin of the operculum) in respect of the other landmarks located on the head (landmarks 1 , $8,12,19$ and 20) and by the relative displacement of landmark 2 (origin of the dorsal fin) towards the anterior-dorsal direction. Besides, in high density group the distance between eyes was higher (landmarks 11 and 12).

There was a general relative posterior body shortening, more intense in high density group, as a consequence of a combined movement of medium-body landmarks (numbers 16, 17, 18) and caudal landmarks (dorsal and ventral origin of the caudal fin, landmarks number 3 and 5 respectively). The former suffered a relative displacement towards the caudal area and the later towards the cephalic area of the body.

Equally, $\mathrm{H}$ fish presented a relative caudal-peduncle enlargement as a consequence of the short displacement localized on landmark 4 (final point of the lateral line) combined with the forward movement of landmarks 3 and 5.

The inclusion of fish sex into the Canonical Variate Analysis did not show different results compared to those obtained without taking sex into account. Accordingly, this variable does not seem to be after the differences found in shape between fish reared under low or high density. Nevertheless, this should be accepted cautiously due to the low number of individuals that formed the experimental groups after separating them according to sex and density treatment. It would be interesting to perform further studies using a similar methodology, to test and quantify whether this species present sexual dimorphism.

Considering that sex did not influence shape, and given that fish used in this experiment presented a very low genetic variability (Sánchez et al. 2005), and that there were not significant differences in water quality among tanks, differences in shape could be attributed to differences in stocking density.

These differences could be the result of social interactions. In general, overlapping at the bottom of tanks stocked with low density of flatfish is lower than in tanks stocked with high density. In this last condition, activity measured as swimming motion, as reported by Duarte et al. (2006), is higher than the one registered in tanks stocked with a lower density of individuals. Nevertheless, more studies about sole behaviour (activity, feeding behaviour, etc) 
in high density, and how it influences shape should be performed to achieve a reliable description of this relationship. Especially interesting would be those experiments conducted to determine a correlation between shape variables and behavioural variables.

Furthermore, note that our results did not indicate a significant size-related shape change, likely due to fish age. Fish under study were adults in the saturating part of its growth trajectory and a suitable determination of size effect on shape change would need a longer experimental time.

In conclusion, to our knowledge this is the first study describing the effect of stocking density on sole shape, specifically at the end of the rearing process, very close to commercialization time. Our results provide a first promising insight in the study of environment-related shape changes in sole. On the other hand, geometric morphometrics has demonstrated a true potential to be incorporated into classical studies of fish quality as a reliable and valuable tool to describe shape changes.

\section{Acknowledgements}

This work was funded by the Spanish Ministerio de Ciencia y Tecnología (MCYT AGL2002-00768)

\section{References}

Barlow, G (1961) Causes and significance of morphological variation in fishes. Syst Zool 10:105-117

Bookstein, F L (1991) Morphometric tools for landmark data: geometry and biology. Cambridge University Press, Cambridge.

Cato, J C (1998) Valuation of seafood safety. Consumer perception of seafood safety and quality. In: Seafood safetyEconomics of hazards analysis and critical control point (HACCP) programmes. FAO Fish Tech Pap 381:11-15

Corti, M, Loy, A, Cataudella, S (1996) Form changes in the sea bass, Dicentrarchus labrax (Moronidae: Teleostei), after acclimation to freshwater: an analysis using shape coordinates. Environ Biol Fish 47:165-175

Duarte, S, Reig, L, Oca, J, Sánchez P, Ambrosio, P P, Flos, R (2006) Behavioural responses of a heterogeneous size sole population at two different stocking densities. In: Abstracts of the EAS and WAS annual meeting, Aqua 2006Linking Tradition and Technology. Higher quality for the consumers, Firenze, Italy. p 266

Engrola, S, Caçao, P, Conceição, L E C, Dinis, M T (2001) Ongrowing of Senegal sole (Solea senegalensis) juveniles at different densities. In: Book of Abstracts of the Aquaculture 2001 WAS Conference, Lake Buena Vista, Florida, USA, 21-25 January 2001

Flos, R, Reig, L, Oca, J, Ginovart, M (2002) Influence of marketing and different land-based systems on gilthead sea bream (Sparus aurata) quality. Aquacult Int 10(1):189-206

Gavaia, P J, Sarasquete, C, Cancela, M L (1999) Determination of mineralized structures in early stages of development of marine teleostei using a modified alcian blue-alizarin red double staining technique for bone and 
cartilage. Biotech Histochem 75(2):79-84

Goodall, C R (1991) Procustes methods in the statistical analysis of shape. J R Statist Soc b 53:285-339

Hammer, $\varnothing$, Harper, D A T, Ryan P D (2001). PAST: Paleontological Statistics Software Package for Education and Data Analyses. Paleontologia Electronica 4(1) art. 4:9pp.

Howell, B R (1998) The effect of stocking density on growth and size variation in cultured turbot, Scophtalmus maximus, and sole, Solea solea. ICES CM L:10

Hubss, C L (1926) The structural consequence of modifications of the developmental rate in fishes, considered in reference to certain problems of evolution. Am Nat 60:57-81

Imsland, A K, Foss, A, Conceiçao, L E C, Dinis, M T, Delbare, D, Schram, E, Kamstra, A, Rema, P, White, P (2003) A review of culture potential of Solea solea and S. senegalensis Rev Fish Biol Fisher 13:379-407

Loy, A, Boglione, C, Cataudella, S (1999) Geometric morphometrics and morpho-anatomy: a combined tool in the study of sea bream (Sparus aurata, sparidae) shape. J Appl Ichthyol 15:104-110

Loy, A, Boglione, C, Gagliardi, F, Ferrucci, L, Cataudella, S (2000) Geometric morphometrics and internal anatomy in sea bass shape analysis (Dicentrarchus labrax L., Moronidae). Aquaculture 186:33-44

Marcus, L F, Bello, E, Garcia-Valdecasas, A (1993) Contributions to morphometrics. Monografías, Museo Nacional de Ciencias Naturales-CSIC. Madrid.

Pavlinov, I Ya, Mikeshina, N G (2002) Principles and methods of geometric morphometrics. J General Biol 63 (6): 473-493. In Russian, with English summary.

Reig, L, Ginovart, M, Flos, R (2003) The application and evaluation of an individual marking technique of sole fingerlings (Solea sp.) for studies of growth. J Appl Ichthyol 19 (1):49-51

Rohlf, F J, Slice, D (1990) Extensions of the Procustes method for the optimal superimpositions of landmarks. Syst Zool 39 (1):40-59

Rohlf, F J, Bookstein, F L (1991) Proceedings of the Michigan Morphometric Workshop, University of Michigan. Special Publication № 2 Ann Arbor.

Rohlf, F J, Marcus, L F (1993) A revolution in morphometrics. Trends Ecol Evol 8: 129-132.

Rohlf, F J (2003) TpsRegr., ver. 1.28. Dept. of Ecology and Evolution, State Univ. of New York at Stony Brook, Stony Brook

Rohlf, F J (2004a) Tps Dig., ver. 1.40. Dept. of Ecology and Evolution, State Univ. of New York at Stony Brook, Stony Brook

Rohlf, F J (2004b) Tps Relw., ver. 1.39. Dept. of Ecology and Evolution, State Univ. of New York at Stony Brook, Stony Brook

Sánchez, P, Viñas, J, Alvarado-Bremer, J, Ambrosio, P P, Flos, R (2005) Mitochondrial control region and microsatellite data show low genetic variability in reared Senegalese sole, Solea senegalensis, preliminary data. In: Howell, B. and Flos, R.(Eds.). Lesson from the past to optimize the future, European Aquaculture Society, Special Publication No. 35, Trondheim, Norway, p 397-398

Schram, E, Van der Heul, J W, Kamstra, A, Verdegem, M C J (2006) Stocking density-dependent growth of Dover sole (Solea solea). Aquaculture 252:339-347 
Taylor, W R, Van Dyke, G C (1985) Revised procedures for staining and clearing small fishes and other vertebrates for bone and cartilage study. Cybium 9 (2):107-119

Zelditch, M L, Swiderski, D L, Sheets, H D, Fink, W L (2004) Geometric morphometrics for biologists: a primer. Elsevier Academic Press, San Diego, USA 